\title{
Minimum amount of penicillin prophylaxis required to control Streptococcus pyogenes epidemic in closed community
}

\author{
A COLLING, IDA KERR, W R MAXTED, JEAN P WIDDOWSON
}

\begin{abstract}
The prophylaxis required to control an epidemic of Streptococcus pyogenes throat infection in a junior detention centre has been reported. In a further epidemic an attempt was made to determine the minimum amount of penicillin required to control the outbreak. Oral penicillin $(0.5 \mathrm{~g})$ given as a single daily dose for 10 days to all boys after entry proved effective.
\end{abstract}

The added risk of relatively deprived adolescent boys developing rheumatic fever is stressed.

\section{Introduction}

We previously described an epidemic of acute tonsillitis associated with Streptococcus pyogenes in a junior detention centre $^{1}$ where boys aged 14-17 stayed for six to eight weeks. During that epidemic swabs were taken from the nose and throat of those who developed acute tonsillitis and routinely from all boys on entry to the centre, again four weeks later, and finally before discharge. At the height of the epidemic $40 \%$ became carriers of group A streptococci and $20 \%$ developed tonsillitis. In a sample of cases, group A haemolytic streptococci were isolated from $31.7 \%$ by the use of dry swabs or unenriched transport medium, but $77 \cdot 6 \%$ yielded positive results with enriched medium duplicate swabs. The most common serotype was M5. Viruses did not appear to be an important cause of acute tonsillitis in this particular epidemic. Serum samples were taken for antibody estimation from 146 boys on entry to the centre, and from 187 carriers together with paired specimens from cases (113). High titres of streptococcal antibodies (antistreptolysin $\mathrm{O}$, anti-desoxyribonuclease $\mathrm{B}$ and anti-M-associated protein) were found not only in cases and carriers but also in boys on entry to the centre. Hygienic measures and the treatment of cases and carriers with penicillin failed to halt the epidemic. Full penicillin prophylaxis was then given to all the boys entering the centre (phenoxymethylpenicillin $0.25 \mathrm{~g}$ four times a day for 10 days), which appeared to be effective in reducing the attack rate. Surprisingly, the epidemic took several years to bring under control. The study began in 1972, but it was early 1977 before the incidence of infection was low enough (reduced from $20.6 \%$ to $4.7 \%$ of new entrants) for us to consider reporting the effectiveness of our methods.

Full penicillin prophylaxis was continued after the epidemic was brought under control and the incidence of tonsillitis remained low during 1977 (figure). Giving penicillin four times a day was very demanding, however, and we relied heavily on the good will of prison officers to muster boys and administer

Kirklevington Detention Centre, Yarm, Cleveland

A COLLING, MD, FRCGP, formerly part-time medical officer IDA KERR, SEN, matron

Division of Hospital Infection, Central Public Health Laboratory, London NW9

W R MAXTED, PHD, FIMLT, top-grade microbiologist (now at Royal Veterinary College, Potters Bar, Herts)

JEAN P WIDDOWSON, BPHARM, PHD, top-grade microbiologist treatment. In early 1978, as a result of national industrial action, prison officers were instructed by their union not to help with medical care. The matron was unable to provide seven-day cover by herself and boys began to receive only intermittent prophylaxis. Since this was unsatisfactory and we had seen so few cases of tonsillitis for two years we decided to stop prophylaxis (18 March, 1978). Almost at once there was a high rate of acute tonsillitis attacks (figure).

We now report on our further clinical surveillance in the same prison from 1977 to 1980 and our attempts to determine the minimum amount of penicillin necessary to control this new epidemic, which developed in 1978.

\section{Materials and methods}

Tonsillitis was diagnosed clinically, using criteria previously described. ${ }^{1}$ Boys who developed acute tonsillitis were treated as before with intramuscular penicillin, $0.6 \mathrm{~g}$ twice a day until symptoms cleared and then oral penicillin, $0.25 \mathrm{~g}$ four times a day for 10 days. Erythromycin $0.25 \mathrm{~g}$ four times a day was given if there was a history of penicillin allergy.

The following prophylactic regimens were used: from 1 Aug 1978 to 30 Nov $19781.0 \mathrm{~g}$ phenoxymethylpenicillin was given in a single dose on day of entry to the centre; from $1 \mathrm{Dec} 1978$ to 31 Aug 1979 $0 \cdot 25 \mathrm{~g}$ in a single dose each day for 10 days after admission; and from 1 Sept 1979 to 31 Aug $19800.5 \mathrm{~g}$ in a single dose each day for 10 days after admission.

\section{Results}

We began by giving penicillin as a single oral dose of $1.0 \mathrm{~g}$ to all boys on entry to the centre, but it was soon apparent that this was having only a partial effect on the high rate of attack (14.7\%). After 16 weeks the dose was increased to $0.25 \mathrm{~g}$ a day for 10 days, a regimen which continued for 40 weeks. The rate of infection fell slightly $(10 \cdot 1 \%)$, but showed no sign of returning to the pre-epidemic basal level. The dose was increased to $0.5 \mathrm{~g}$ a day for 10 days, resulting in a gradual decline to a very low rate $(2 \cdot 1 \%$ ) (see figure). Two boys developed acute rheumatism (Jones's criteria) during the study period.

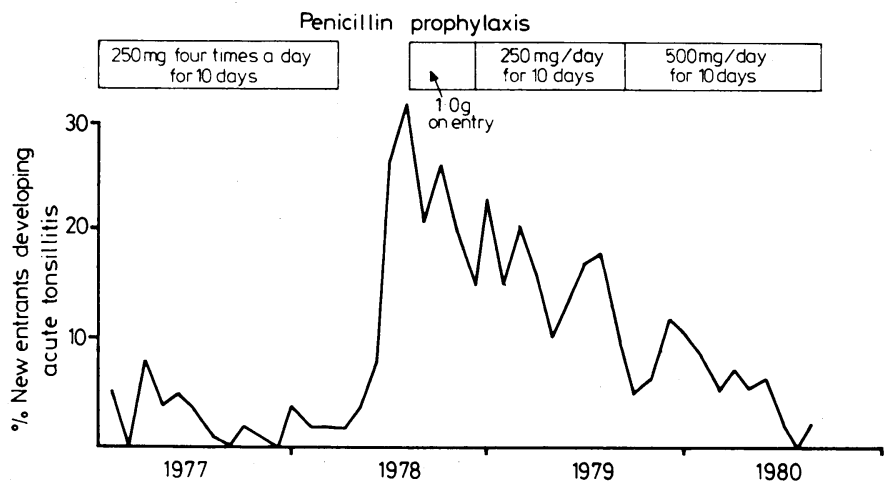

Percentage of new entrants to Kirklevington Junior Detention Centre developing acute tonsillitis. 


\section{Discussion}

From our previous work we knew that the epidemic of tonsillitis we described was not controlled by the treatment of cases and carriers alone and that full penicillin prophylaxis to all boys on entry was essential. This regimen entailed giving penicillin four times a day; this was difficult to administer and was stopped in 1978 , when the epidemic seemed to be under control. Though these measures appeared to be effective, we were unable to claim with certainty that the improvement was anything more than fortuitous. A further epidemic began within eight weeks of stopping the prophylaxis (March 1978), which made it more likely that the control we had reported had resulted from penicillin prophylaxis.

This new epidemic presented an opportunity to try to determine the minimum amount of prophylactic penicillin required for control. From our results $0.5 \mathrm{~g}$ oral phenoxymethylpenicillin given daily to all boys for 10 days after entry seemed effective; when administered at breakfast and before dispersal to their various activities this was not unduly disruptive.

Four cases of rheumatic fever occurred in our first survey and there were two further cases during this epidemic. Since the incidence of rheumatic fever in the United Kingdom is now believed to be very low, these attacks highlight the need to control epidemics of tonsillitis in closed communities.

Once again we found a sluggish response to prophylaxis. From our previous experience, perhaps we should have foreseen that it would take time to bring the epidemic under control. We do not know whether we could have achieved a quicker response by treating all boys who were in the centre at the height of the epidemic with oral penicillin, but this would seem likely.

There may well be a difference between controlling epidemics in closed communities, such as detention centres, which have a changing population, and semi-closed communities, such as boarding schools, which have a more fixed population. Gastanaduy et $\mathrm{al}^{2}$ recently reported an epidemic in a semi-closed community of 300 people that was brought under control in three or four months by the administration of intramuscular penicillin or oral antibiotics (phenoxymethylpenicillin or erythromycin) to cases and carriers.

We have already suggested that adolescent boys may be particularly prone to Streptococcus pyogenes infections. Bringing together boys of this age group into closed communities will compound the problem, especially if the population is always changing. We do not have figures from other detention centres, but we are told that sore throats are very common. We suspect that our experience at Kirklevington is not unique and in the relatively socially deprived group of boys in detention centres the risk of developing rheumatic fever may well be higher than in the general population.

\section{References}

${ }^{1}$ Colling A, Kerr I, Maxted WR, Widdowson JP. Streptococcal infection in a junior detention centre: a five-year study. $\mathcal{F}$ Hyg (Camb) $1980 ; 85$ : 331-41.

2 Gastanaduy AS, Kaplan EL, Huwe BB, McKay C, Wannamaker LW. Failure of penicillin to eradicate group A streptococci during an outbreak of pharyngitis. Lancet 1980;ii:498-501.

(Accepted 24 May 1982)

\section{SHORT REPORTS}

\section{Do patients receiving regular haemodialysis need folic acid supplements?}

Patients receiving regular haemodialysis often receive folic acid supplements because foods rich in this vitamin often need to be restricted and loss across the dialysis membrane is likely to occur because of its low molecular weight and only moderate protein binding. ${ }^{12}$ We have shown that the loss of folic acid during dialysis is equivalent to a daily loss only slightly greater than the urinary loss in normal subjects, ${ }^{3}$ and in a retrospective study we found no evidence of folate deficiency in 72 patients who had received haemodialysis for a mean of 49 months. ${ }^{3}$ We now report the results of a double-blind placebo-controlled crossover study to investigate the change in haemoglobin concentration and mean corpuscular volume during six months of treatment with folic acid.

\section{Patients, methods, and results}

Twenty-nine patients ( 16 men, 13 women) aged 18-59 (mean 42) who had received regular haemodialysis for nine to 143 (mean 69) months, entered the study. All were taking iron supplements (Ferrogradumet) orally once daily and none had received folic acid, cobalt chloride, androgens, or a blood transfusion within the preceding six months. The patients were divided into two groups of similar size matched for age, sex, and haemoglobin concentration. Group 1 received folic acid $5 \mathrm{mg}$ daily and group 2 a matching placebo. After six months the groups crossed over, group 1 receiving placebo and group 2 folic acid for a further six months. Haemoglobin concentration and mean corpuscular volume were measured monthly by using a Coulter counter. Serum and red cell folate concentrations were measured at entry and at six and 12 months using a competitive binding assay (Becton Dickinson folate radioassay kit). The patients were seen monthly and completed a questionnaire regarding symptoms and blood loss, grading them as "none," "slight," or "troublesome" (scored 0,1 , or 2 in the analysis).

The significance of changes in haematological variables was measured using non-parametric regression analysis.
Six patients failed to complete the 12 months' trial. Two were withdrawn for transfusion, one after five months' treatment with placebo and the other after six months' treatment with folic acid. Four further patients were withdrawn while taking placebo; one because of sleeplessness, one headache and nausea, one penile irritation, and one worsening psoriasis. Red cell and serum folate concentrations were normal in all patients at entry. They rose significantly during treatment with folic acid and not during placebo periods, confirming compliance with trial medication. The order in which the treatments were given did not appear to contribute to the treatment effect. Blood loss reported during the treatment periods was similar. The mean haemoglobin concentration on entry was $8.0 \mathrm{~g} / \mathrm{dl}$. During treatment with folic acid there was a mean fall of $0.38 \mathrm{~g} / \mathrm{dl}$ and a mean rise during placebo treatment of $0.25 \mathrm{~g} / \mathrm{dl}$. This difference is not significant $(p>0.05)$. The $95 \%$ confidence limits for the difference between folic acid and placebo are -1.49 and $0 \cdot 23$, corresponding to a fall of $1.49 \mathrm{~g} / \mathrm{dl}$ on folate to a fall of 0.23 $\mathrm{g} / \mathrm{dl}$ on placebo. Changes in mean corpuscular volume did not differ significantly. Symptoms of nausea, headache, vivid dreams, agitation, and irritability were slightly more common during treatment with folic acid, and anorexia, blurred vision, and sleep disturbance more common during treatment with placebo. None of these differences approached significance. The results were essentially unaltered by including data from the six patients withdrawn from the trial.

\section{Comment}

This study has shown no benefit from six months' treatment with folic acid and indeed a slight fall in haemoglobin concentration occurred during treatment with folic acid. In addition, there was a weak association with nausea, headache, vivid dreams, agitation, and irritability, symptoms that have been ascribed to folic acid previously. ${ }^{4}$ We therefore see no justification for the use of folic acid supplements for adequately nourished patients receiving regular haemodialysis.

${ }^{1}$ Johns DG, Sperti S, Burgen ASV. The metabolism of tritiated folic acid in man. F Clin Invest 1961;40:1684-95.

2 Soliman HA, Loesen H. Folic acid binding by human plasma albumin. Scand $\mathcal{F}$ Clin Lab Invest 1976;36:299-304.

${ }^{3}$ Cunningham J, Sharman VL, Goodwin FJ, Marsh FP. Do patients receiving haemodialysis need folic acid supplements ? Br Med $\mathcal{f} 1981 ; 282$ 1582. 